\title{
Robots and Artificial Intelligence
}

$\mathrm{R}$ obots are programmable machines that are usually designed to carry out a series of actions autonomously. They can be programmed to do repetitive or labor-intensive jobs that would readily exhaust humans, or to carry out particular tasks under the circumstances that are dangerous to humans. Artificial intelligence (AI) is a branch of computer science. It involves developing computer programs to complete tasks that would otherwise require human intelligence. $\mathrm{Al}$ is, in essence, a bunch of algorithms that can tackle learning. perception, problem-solving, language-understanding and/or logical reasoning. Though robotics and $\mathrm{Al}$ are two different areas, they are not mutually exclusive. It is conceivable that robots are more powerful when they are equipped with an artificially intelligent brain.

CAS scientists have made many landmark accomplishments in robots and X. as well as artificially intelligent robots.

In 1995. scientists at the (CS Shenyang Institute of Automation (SIA) took the lead in developing a cableless autonomous underwater robot (CR-01) that can work at 6,000-meter depth. It took part in the two investigations of the Pacific Ocean in 1995 and 1997. enabling China have the ability to conduct a detailed exploration of the sea areas except the trench. This achievement also enables (hina to rank among the top countries in this regard in the world. winning the first prize of the National Science and Technology Progress Award in 1998.

Later in 2012. SIA scientists independently developed China's first 6.000-meter unmanned cableless autonomous underwater vehicle named Oianlong-1. capable of automatic orientation, vertical movement. lateral movement and remote navigation among many others. Two years later. they designed a new model Oianlong-2 with high intelligent autonomous obstacle avoidance ability and stable navigation control ability. proclaiming that China s underwater autonomous robot technology has reached the international advanced level.

Besides, the industrial robot technology developed by the SII scientists have been successfully commercialized. $A$ derivative company called SILSUN Robots have earned a leading share of the global market in mobile robots. In recent vears. SIA scientists have also developed many special robots such as polar scientific research mobile robots, rotor flying robots. nanomanipulation robots, ultra-high voltage line inspector, anti-terrorism explosion-proof robots, ete.

In 2013. scientists at the CaS Hefei Institute of Physical Science (CASIIIPS) independently developed China's first humanoid robot lugong that is capable of

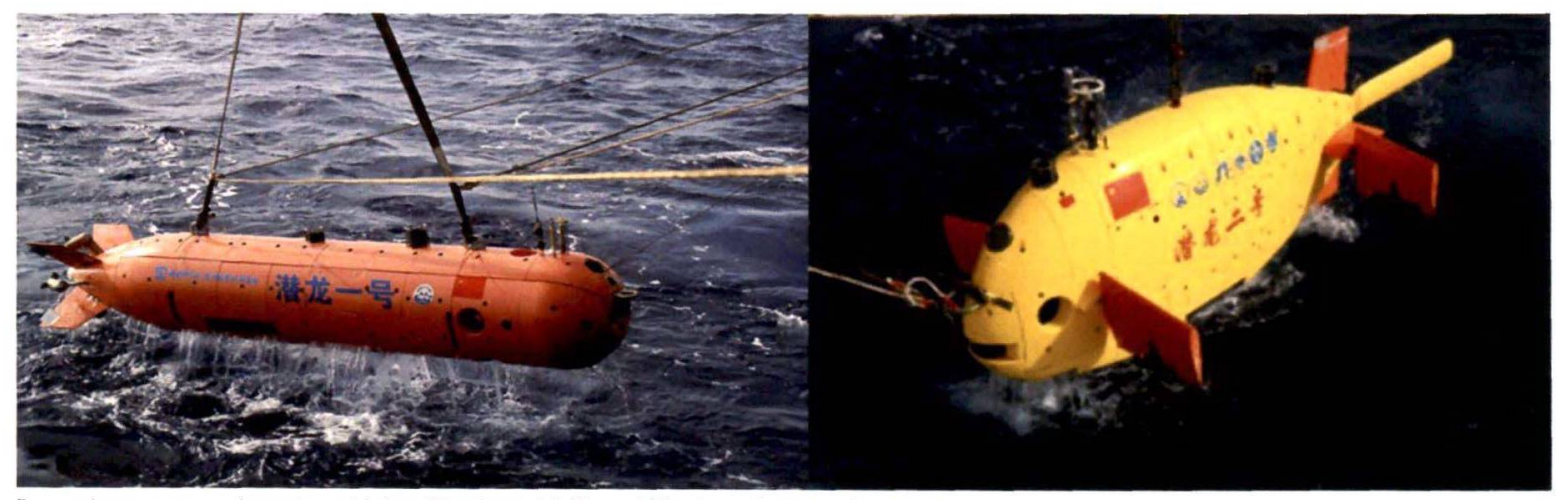

Two autonomous underwater vehicles, Qian/ong-1 (left) and Qian/ong-2 (right). (Credit: SIA) 


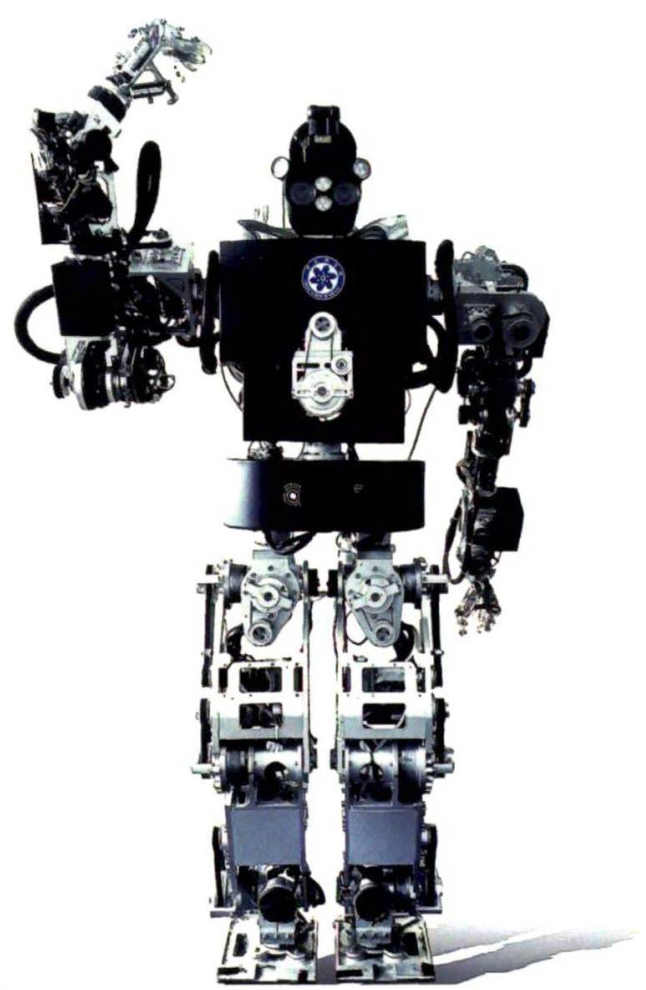

China's first humanoid robot Yugong. (Credit: CASHIPS)

walking and multitasking under complex situations.

In the field of AI, Hangwang Technology, a company hatched from the CAS Institute of Automation (CASIA), created the world's first large-character set handwritten Chinese character input system in 1999. Its technology of handwritten Chinese character recognition won the first prize of National Science and Technology Progress Award in 2001. Scientists at the CASIA also developed many other technologies with wide applications such as iris recognition, face recognition, voice recognition. intelligent video surveillance, molecular imaging. etc. They also developed the M program of 'CASLA-

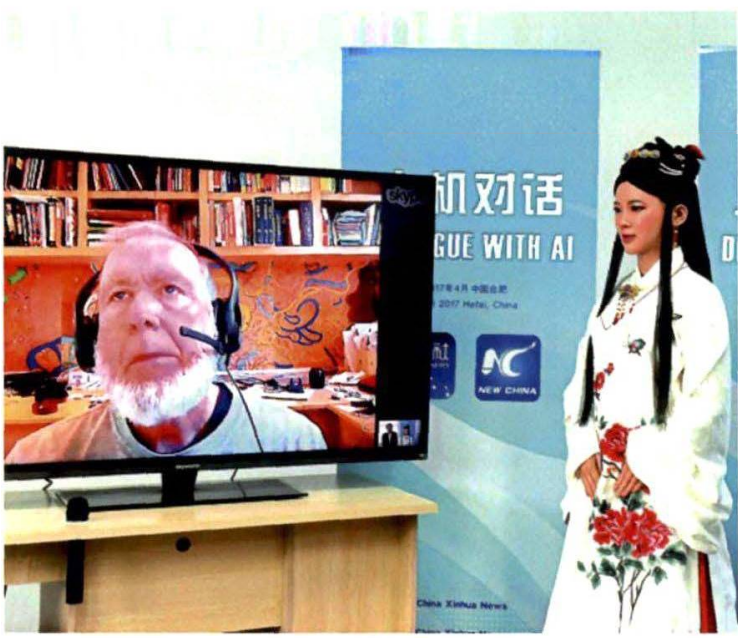

China's first experience interactive robot Jiajia is equipped with the iFlytek's intelligent voice interaction technology (Credit: CAS)

Prophet $1.0^{\prime}$ and the bionic robot fish, which are both of important applications.

In addition, scientists at the CAS Institute of Computing Technology (ICT) made breakthroughs in the field machine translation. In 1992, they successfully developed an intelligent English-Chinese machine translation system IMT/EC (IMT/863), winning the first prize of the National Science and Technology Progress Award in 1995

Research advances made by scientists from the University of Science and Technology of China (USTC) hatched iFlytek, a pioneer in China's voice recognition and interaction industry and a global leader in the field of AI. iFlytek was ranked $6^{\text {th }}$ on the MIT Technology Review's list of the World's Top50 Smartest Companies in 2017. It has set world records for many times in global top-level competitions and authoritative evaluations.

The merging of robots and $\mathrm{AI}$ is on its way, heralding the advent of new exciting techniques that would change our ways of life at a larger scale. 